\title{
Self-assembled quantum dots in a nanowire system for quantum photonics
}

\author{
M. Heiss' ${ }^{1 \dagger}$, Y. Fontana ${ }^{1 \dagger}$, A. Gustafsson' ${ }^{2}$, G. Wüst ${ }^{3}$, C. Magen ${ }^{4}$, D. D. O'Regan ${ }^{5}$, J. W. Luo ${ }^{6}$, \\ B. Ketterer', S. Conesa-Boj' ${ }^{1}$ A. V. Kuhlmann ${ }^{3}$, J. Houel ${ }^{3}$, E. Russo-Averchi' ${ }^{1}$, J. R. Morante ${ }^{7,8}$, \\ M. Cantoni ${ }^{9}$, N. Marzari ${ }^{5}$, J. Arbiol ${ }^{10}$, A. Zunger ${ }^{11}$, R. J. Warburton ${ }^{3}$ and A. Fontcuberta i Morral ${ }^{1 \star}$
}

\begin{abstract}
Quantum dots embedded within nanowires represent one of the most promising technologies for applications in quantum photonics. Whereas the top-down fabrication of such structures remains a technological challenge, their bottom-up fabrication through self-assembly is a potentially more powerful strategy. However, present approaches often yield quantum dots with large optical linewidths, making reproducibility of their physical properties difficult. We present a versatile quantum-dot-innanowire system that reproducibly self-assembles in core-shell GaAs/AIGaAs nanowires. The quantum dots form at the apex of a GaAs/AIGaAs interface, are highly stable, and can be positioned with nanometre precision relative to the nanowire centre. Unusually, their emission is blue-shifted relative to the lowest energy continuum states of the GaAs core. Large-scale electronic structure calculations show that the origin of the optical transitions lies in quantum confinement due to Al-rich barriers. By emitting in the red and self-assembling on silicon substrates, these quantum dots could therefore become building blocks for solid-state lighting devices and third-generation solar cells.
\end{abstract}

S emiconductor quantum dots have been shown to be excellent building blocks for quantum photonics applications, such as single-photon sources and nano-sensing. Desirable properties of a single-photon emitter include high-fidelity anti-bunching (very small $g^{2}(t=0)$ ), narrow emission lines (ideally transform limited to a few microelectronvolt) and high brightness $(>1 \mathrm{MHz}$ count rate on standard detector). For simplicity, these properties should be achieved either with electrical injection or non-resonant optical excitation. Desirable properties of a nano-sensor include a high sensitivity to local electric and magnetic fields, with the quantum dot located as close as possible to the target region. A popular realization involves Stranski-Krastanow InGaAs quantum dots embedded in a three-dimensional matrix, which are excellent building blocks for the realization of practical singlephoton sources ${ }^{1}$. However, the photon extraction out of the bulk semiconductor is highly inefficient on account of the large mismatch in refractive indices of GaAs and vacuum. An attractive way forward is to embed the quantum dots in a nanowire ${ }^{2}$. To solve the extraction problem, the nanowire is designed to operate as a single-mode waveguide, a so-called photonic nanowire, with a taper as photon out-coupler ${ }^{3}$. Also, for nano-sensing applications, a quantum dot in a nanowire can be located much closer to the active medium. Top-down fabrication of the photonic waveguide is technologically complex, however. Bottom-up fabrication of the photonic waveguide is very attractive ${ }^{4-6}$, but it is at present challenging to self-assemble quantum dots in the nanowires with narrow linewidths and high yields ${ }^{7,8}$. Nano-sensing applications are at present not highly developed. Other degrees of freedom of the quantum-dot-in-nanowire system that can be usefully exploited are the mechanical modes for optomechanics, and doping for $\mathrm{p}-\mathrm{n}$ junctions with applications in light harvesting ${ }^{9,10}$.

Here we present a promising new quantum-dot-in-nanowire system. A schematic of the physical structure is shown in Fig. 1a. The structure consists of Al-poor $\mathrm{Al}_{x} \mathrm{Ga}_{1-x} \mathrm{As}(x \sim 10 \%)$ quantum dots in an Al-rich $\mathrm{Al}_{x} \mathrm{Ga}_{1-x} \mathrm{As}(x \sim 60 \%)$ barrier wrapped in an intermediate Al-content matrix $(x \sim 33 \%)$. The quantum dots form in the ridge of an AlGaAs nanowire. The self-assembly is driven by the different $\mathrm{Ga}$ and $\mathrm{Al}$ adatom mobilities on the nanowire surface, leading to $\mathrm{Al}$ segregation. The quantum dots can be positioned close to the nanowire surface or close to the nanowire core during the growth simply by choosing the growth mode, lateral or radial, and the overall diameter of core and shell. We note that the quantum dot size is independent of the core diameter. Significantly, the nanowire growth is not complicated by fluctuations in crystal structure (polytypism). We find that the quantum dots are very stable, surviving prolonged electron beam bombardment, exposure to air and so on, and that the quantum-dot-in-nanowire growth is very reproducible from one run to the next: there is a wide window of parameters under which they form. The quantum dots have excellent optical properties even when they are located just a few nanometres from the surface: individual quantum dots are very bright (Megahertz count rate) even without engineering the

\footnotetext{
${ }^{1}$ Laboratoire des Matériaux Semiconducteurs, École Polytechnique Fédérale de Lausanne, 1015 Lausanne, Switzerland, ${ }^{2}$ Solid State Physics, The Nanometer Consortium, Lund University, Box 118, Lund S-221 00, Sweden, ${ }^{3}$ Department of Physics, University of Basel, Klingelbergstrasse $82, \mathrm{CH} 4056 \mathrm{Basel}$, Switzerland, ${ }^{4}$ Instituto de Nanociencia de Aragon-ARAID and Departamento de Física de la Materia Condensada, Universidad de Zaragoza, 50018 Zaragoza, Spain, ${ }^{5}$ Theory and Simulation of Materials (THEOS), École Polytechnique Fédérale de Lausanne, 1015 Lausanne, Switzerland, ${ }^{6}$ National Renewable Energy Laboratory, Golden, Colorado 80401, USA, ${ }^{7}$ Catalonia Institute for Energy Research, IREC. 08930 Sant Adrià del Besòs, Spain, ${ }^{8}$ Department dÉlectrònica, Universitat de Barcelona, 08028 Barcelona, Spain, ${ }^{9}$ Interdisciplinary Center for Electron Microscopy, École Polytechnique Fédérale de Lausanne, 1015 Lausanne, Switzerland, ${ }^{10}$ Institució Catalana de Recerca i Estudis Avançats (ICREA) and Institut de Ciència de Materials de Barcelona, ICMAB-CSIC, E-08193 Bellaterra, CAT, Spain, ${ }^{11}$ University of Colorado, Boulder, Colorado 80309, USA. "These authors contributed equally to this work. *e-mail: anna.fontcuberta-morral@epfl.ch.
} 
a

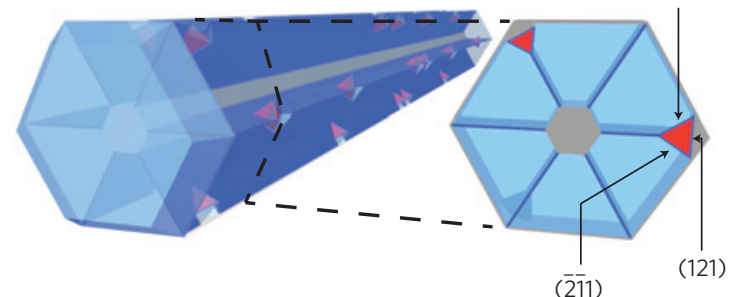

(21)

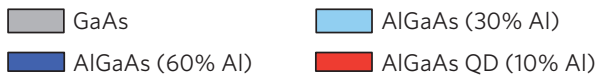

b

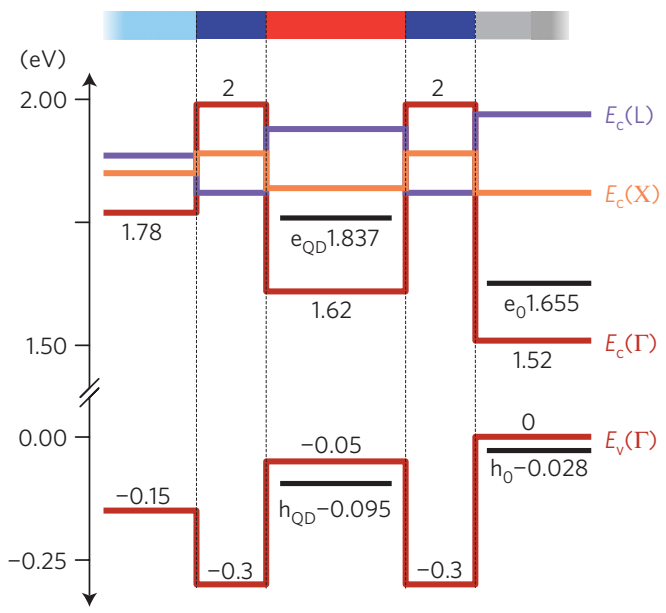

Figure 1 | Schematics of the quantum-dot-in-nanowire system.

a, The nanowire consists of GaAs. The quantum dot forms close to the outer edge of an $\mathrm{Al}_{x} \mathrm{Ga}_{1-x} \mathrm{As}$ shell. Aluminium segregates at the nanowire edges owing to the lower mobility of aluminium. At the outer edge of the $\mathrm{Al}_{x} \mathrm{Ga}_{1-x}$ Aslayer, $\mathrm{Al}$ segregates further in the [112] directions, leading to $\mathrm{Al}$ depletion and the formation of a nanoscale inclusion, an Al-poor $A I_{x} \mathrm{Ga}_{1-x}$ As quantum dot. $\mathbf{b}$, The band edge diagram showing from left to right the $\mathrm{Al}_{x} \mathrm{Ga}_{1-x} \mathrm{As}$ matrix and barriers, the lowest energy states confined to the quantum dot and the external GaAs capping taken from atomistic pseudopotential theory.

photonic modes, the linewidths are small (sub-100 $\mu \mathrm{eV}$ ) and the photons are highly anti-bunched (the upper bound on $g^{2}(t=0)$ is just 2\%) even with intense non-resonant excitation.

An unusual feature is the blue-shift of the quantum dot emission relative to emission from electrons and holes in the lowest energy continuum states, in this case emission from the GaAs substrate, the core. This wavelength ordering of quantum dot and continuum emission is reversed relative to Stranski-Krastanow quantum dots. We interpret this unusual result with large-scale calculations using both the empirical pseudopotential method (modelling explicitly 500,000 atoms in this dot-in-wire structure) and density functional theory (modelling a wire geometry with up to 12,000 atoms). The calculations show that whereas the states at the band edge of the system as a whole are indeed located in the GaAs layers, states at higher energy exist, confined to the quantum dot. The results are summarized in an energy level diagram, Fig. $1 \mathrm{~b}$, which shows the band edge valence and conduction states, $\mathrm{h}_{0}$ and $\mathrm{e}_{0}$, and the quantum dot valence and conduction states, $\mathrm{h}_{\mathrm{QD}}$ and $\mathrm{e}_{\mathrm{QD}}$. For Stranski-Krastanow dots, the continuum states are associated with the wetting layer, a thin 2D layer connecting the quantum dots, and lie at higher energy than the lowest energy quantum dot states, $\mathrm{e}_{0}$ and $\mathrm{h}_{0}$. For the quantum-dot-in-nanowire system presented here, this energy reversal of quantum dot and continuum emission represents a new Ansatz for a solid-state single-photon emitter.
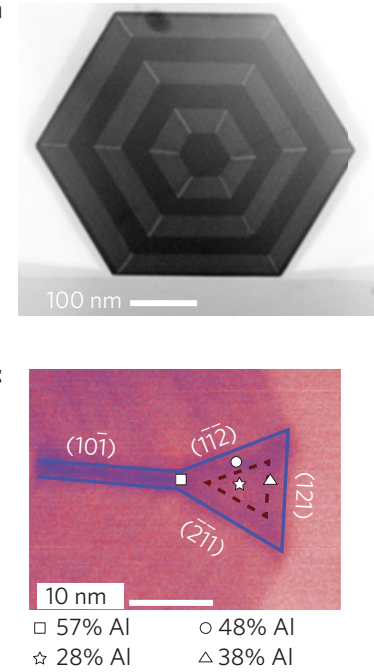

e

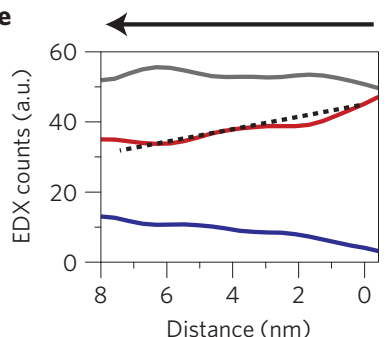

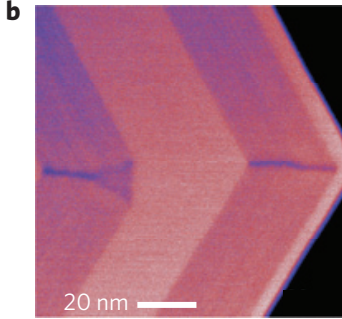

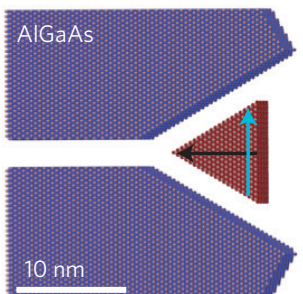

$\mathbf{f}$

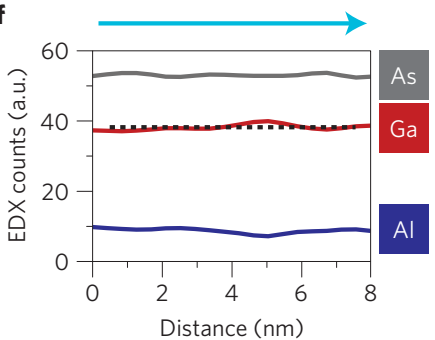

Figure 2 | Structure of quantum-dots-in-a-nanowire.

a, Aberration-corrected high-angle annular dark-field STEM image of the entire cross-section of a GaAs nanowire coated with multiple $\mathrm{Al}_{0.33} \mathrm{Ga}_{0.77} \mathrm{As} / \mathrm{GaAs}$ shells. b, Zoom-in of a. c, Detail of the Al-poor quantum dot located within the fork-like Al-rich stripes. The colouring has been chosen to enhance the contrast between the different regions. d, Three-dimensional atomic model of the cross-sectional STEM image shown in c. The different colours have been introduced for clarity. e,f, Chemical profiling of a single quantum-dot-in-nanowire using EDX spectroscopy along two orthogonal directions.

For Stranski-Krastanow dots, the wetting layer creates problems on non-resonant excitation: it can emit strongly ${ }^{11}$ and trap charges over times comparable to the radiative lifetime ${ }^{12}$, resulting in an increase in $g^{2}(t=0)$ as the pump power is increased ${ }^{13}$. These problems are bypassed here. This, along with the high material quality, is responsible for the very high fidelity photon anti-bunching in the emission from a single quantum-dot-in-nanowire.

GaAs nanowires were grown by molecular beam epitaxy on a 2 inch $\operatorname{Si}(111)$ substrate using the gallium-assisted method ${ }^{14}$. After stopping the axial growth, the conditions were switched from axial to lateral to grow $\mathrm{Al}_{x} \mathrm{Ga}_{1-x}$ As shells with $\mathrm{Al}$ compositions of $x=33 \%$ and $x=51 \%$ (refs 15,16). In the lower $\mathrm{Al}$ content shell, we also alternated the $\mathrm{Al}_{x} \mathrm{Ga}_{1-x}$ As shells with $20 \mathrm{~nm}$ thick GaAs quantum wells. The nanowires were characterized structurally in crosssection using high-angle annular dark field scanning transmission electron microscopy (STEM). Images of a GaAs nanowire covered with alternating layers of $\mathrm{Al}_{0.33} \mathrm{Ga}_{0.77} \mathrm{As}$ and $\mathrm{GaAs}$ are shown in Fig. 2a-c. The lighter regions correspond to GaAs and the darker regions to $\mathrm{Al}_{x} \mathrm{Ga}_{1-x} \mathrm{As}$, with the contrast correlating directly with the Al content (Z-contrast). We observe the formation of dark stripes at the nanowire corners, indicating $\mathrm{Al}$ enrichment. This accumulation is consistent with the difference in chemical potential and adatom mobility on (110) and (112)-type facets ${ }^{17-20}$. More intriguing is the morphology of some stripes at the end of the $\mathrm{Al}_{x} \mathrm{Ga}_{1-x} \mathrm{As}$ layer (Fig. 2b,c). The stripes open up, leaving a region of a few 

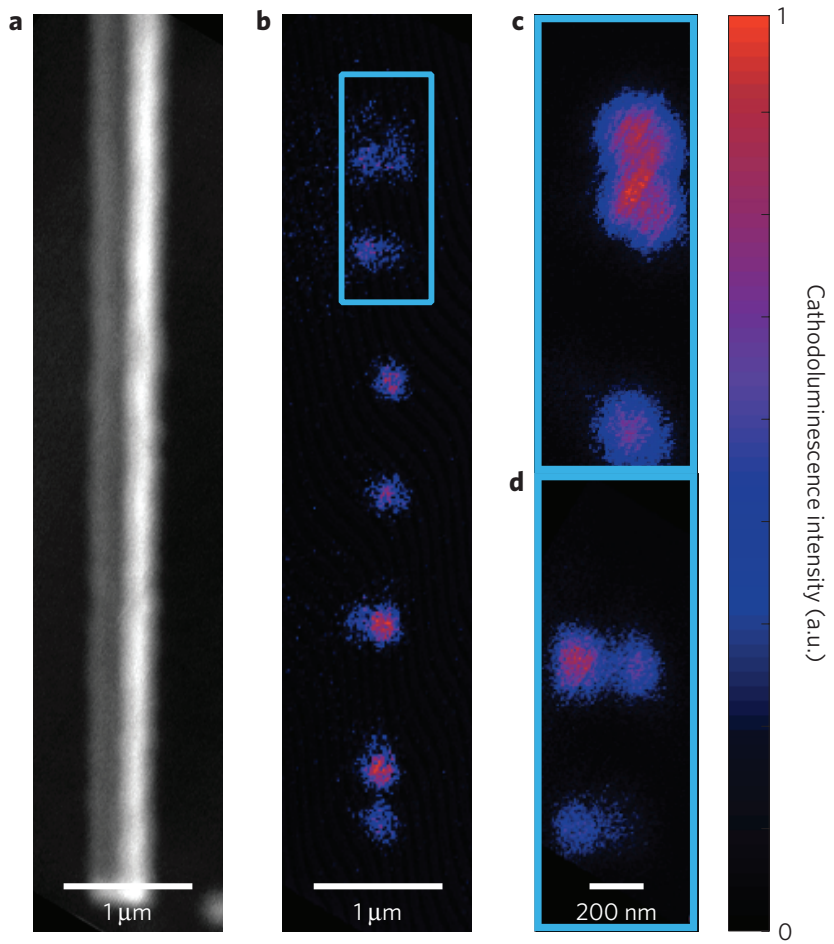

Figure 3 | Cathodoluminescence of a single nanowire. a, Electron microscopy image of a GaAs nanowire with a $\mathrm{Al}_{0.75} \mathrm{Ga}_{0.25} \mathrm{As}$ shell. b. Cathodoluminescence mapping of a nanowire detecting emission at $677 \mathrm{~nm}$. c,d, Detailed cathodoluminescence map showing spatially localized cathodoluminescence centres corresponding to quantum dots less than $200 \mathrm{~nm}$ apart on two adjacent edges of the nanowire emitting at $677 \mathrm{~nm}$.

nanometres in extent with low $\mathrm{Al}$ content: this region constitutes the quantum dot. Figure $2 \mathrm{c}$ shows how the Al-rich stripe following the (10i) plane bifurcates into two Al-rich stripes parallel to the $(1 \overline{1} \overline{2})$ and $(\overline{2} \overline{1} 1)$ planes, forming a Y-like shape, terminated on a polar (121) plane. Chemical profiling realized by energy-dispersive X-ray (EDX) spectroscopy along the two principal directions of the quantum dot is shown in Fig. 2e,f. In the direction from the quantum dot base towards the apex, the Ga signal decreases and the Al signal increases, whereas across the base, the $\mathrm{Ga}$ and $\mathrm{Al}$ signals remain constant. This information is consistent with a shape in the form of a pyramid, as described in the Supplementary Information.

We have probed the optical functionality of these novel quantum dots with both cathodoluminescence (Fig. 3) and photoluminescence (Fig. 4). In both cases, in addition to a broadband emission around $820 \mathrm{~nm}(1.51 \mathrm{eV})$, which we attribute to emission from the GaAs core, we observe sharp emission lines at higher energy, in the red part of the spectrum, Fig. 4a. Representative cathodoluminescence measurements in the spectral region $630-690 \mathrm{~nm}$ (1.97-1.80 eV) along with the corresponding electron microscopy image are shown in Fig. 3. Whereas the $820 \mathrm{~nm}$ cathodoluminescence is spatially continuous (see Supplementary Information), the $677 \mathrm{~nm}$ cathodoluminescence is spatially discontinuous along the nanowire (Fig. $3 \mathrm{~b}-\mathrm{d}$ ): there is a chain of bright, nanoscale emitters. This suggests that the quantum dots identified in the structural characterization are indeed responsible for the optical emission in the red. This is reinforced by images at higher magnification (Fig. 3c,d), which show extremely localized emitters separated laterally by less than $200 \mathrm{~nm}$, corresponding to quantum dots on adjacent edges of the nanowire.

Further confirmation that the structures identified in the TEM analysis are optically active comes from photoluminescence charac- terization of individual quantum dots. The photoluminescence was collected in a side-on geometry and its polarization dependence was measured as a probe of the dielectric environment. The broadband emission from the core is strongly polarized along the axis of the nanowire, reflecting the pronounced dielectric anisotropy (Fig. 4b). However, the sharp photoluminescence lines from individual quantum dots are preferentially polarized in quantum-dot-dependent directions and, in some cases, the polarization lies in a direction perpendicular to the nanowire axis (Fig. 4b). This observation supports the assignment of the sharp quantum dot photoluminescence lines to emitters located not on the nanowire axis but close to the surface.

A zoom-in of a typical photoluminescence spectrum of a single quantum dot is depicted in Fig. 4c. For this particular quantum dot, the emission is centred at $676 \mathrm{~nm}(1.83 \mathrm{eV})$, with a full-width at half-maximum (FWHM) of $36 \mu \mathrm{eV}$. The linewidth varies from dot to dot. The smallest linewidth observed so far is $\sim 29 \mu \mathrm{eV}$, with most quantum dots showing sub- $100 \mu \mathrm{eV}$ linewidths. The single quantum dot photoluminescence is very bright: the count rate on our single-photon detector is $\sim 2 \mathrm{MHz}$ at saturation (see Supplementary Information). A decay curve of the single quantum dot emission following pulsed excitation is also shown in the Supplementary Information. The decay is described by a single exponential over three decades. Given the brightness of the quantum dots, the physical mechanism of this decay must be spontaneous emission, with the associated lifetime, $\tau=450 \pm 20 \mathrm{ps,}$ representing the radiative decay time. In other words, once an electron-hole pair is created in the quantum dot, it decays by spontaneous emission and not by electron and hole relaxation to the band edge states, $\mathrm{e}_{0}$ and $\mathrm{h}_{0}$. The value of $\tau$ is sub-nanosecond, consistent with a large electron-hole overlap ${ }^{21}$, that is, inconsistent with a transition involving an electron and hole localized in different regions of space: both electron and hole are confined to the quantum dot.

To determine the quantum character of the emission, namely, the nature of the anti-bunching, we have measured the timedependence of the second-order intensity correlation $g^{2}(t)$ with a Hanbury Brown-Twiss interferometer. $g^{2}(t=0)$ characterizes the fidelity of the anti-bunching. Furthermore, with cw excitation, the full $g^{2}(t)$ function is sensitive to the dynamics, with two-level and three-level systems behaving very differently. This allows us to probe if the quantum-dot-in-nanowire behaves like a two-level atom for which $g^{2}(t)=1-\exp (-|t| / \tau)$. An example histogram with $\mathrm{cw}$ excitation is shown in Fig. $4 \mathrm{~d}$. There is a very clear dip at time delay zero $(t=0)$, demonstrating anti-bunching in the photon statistics. To probe two-level behaviour, we take $\tau$ from the lifetime measurement, and we measure in situ the temporal response function of the experimental set-up (see Supplementary Information) to quantify the jitter (dominated by the detectors). We then calculate the convolution of the two-level atom result with the system response. Figure $4 \mathrm{~d}$ shows that this procedure describes the experimental results extremely well. We stress that this method does not involve any fit parameters; in fact, the agreement cannot be improved by allowing $\tau$ to vary in a fit procedure. In this way, we find that the upper bound on the true value of $g^{2}(t=0)$ is $2 \%$ : the fidelity of the photon anti-bunching is extremely high. This result is corroborated by measurements of $g^{2}(t=0)$ using a pulsed laser source. However, as a more significant and general statement, we find that this quantum-dot-in-nanowire mimics a two-level atom very closely.

The origin of these two-level-atom-like optical properties is, at first sight, not so obvious based on a comparison with the well-known Stranski-Krastanow dots. In a conventional StranskiKrastanow InGaAs quantum dot embedded in a GaAs matrix, the GaAs has the highest energy band edges, higher than the InGaAs quantum dot, and higher too than the wetting layer which forms an intermediate $2 \mathrm{D}$ energy band. Photoexcitation of the wetting layer 

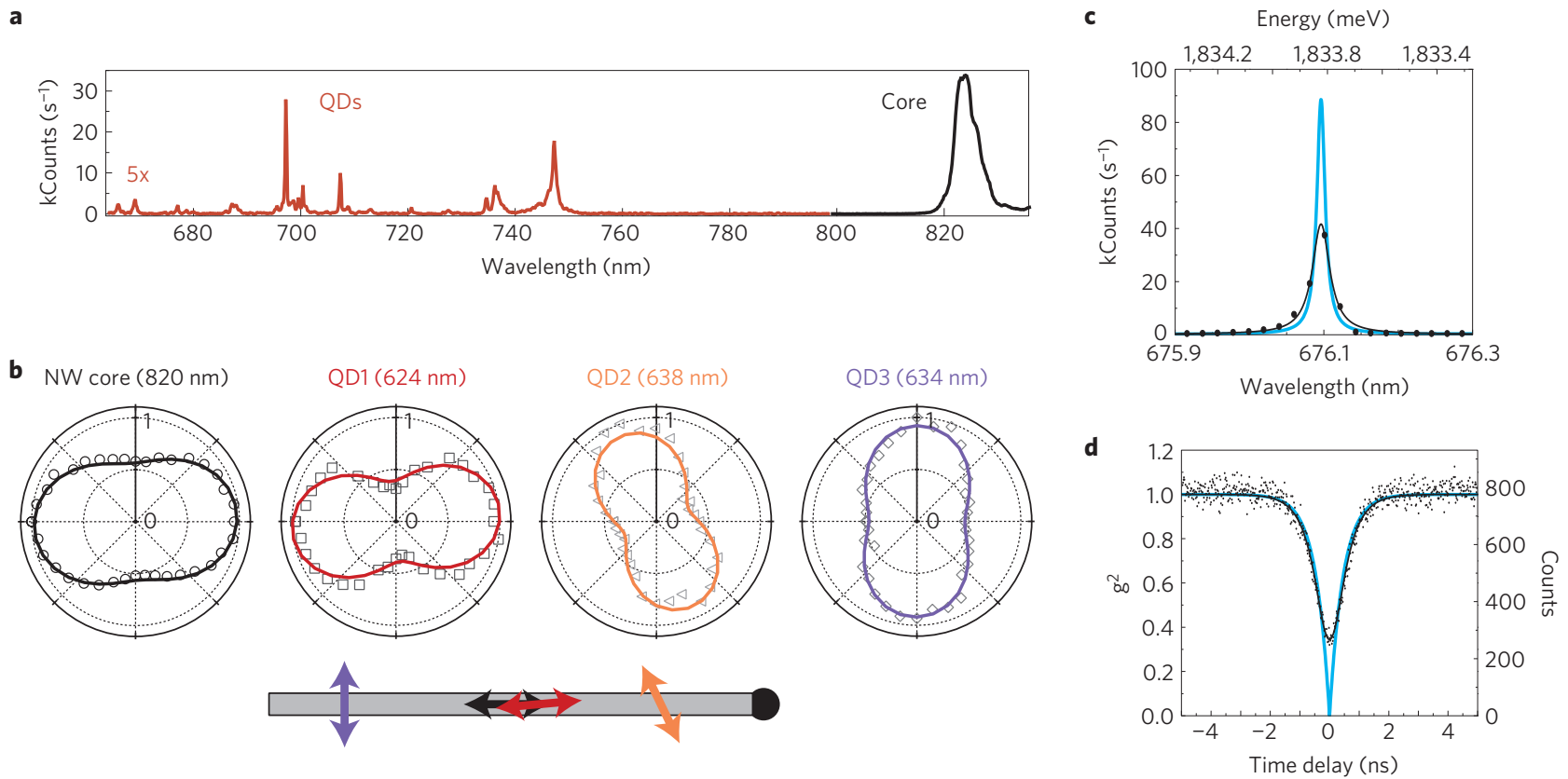

Figure 4 | Photoluminescence of a quantum-dot-in-nanowire system. a, Photoluminescence recorded from a nanowire with non-resonant excitation at $488 \mathrm{~nm}$. The broad peak at $820 \mathrm{~nm}$ arises from emission from the GaAs core; the sharp peaks at shorter wavelength arise from the quantum dots. b. Azimuthal polarization analysis of the emission from the nanowire core and from three quantum dots. c shows a photoluminescence spectrum at a sample temperature $4.2 \mathrm{~K}$ with $0.03 \mu \mathrm{W} \mu \mathrm{m}^{-2}$ with an excitation wavelength of $632.8 \mathrm{~nm}$. The points are the measured counts on the

charge-coupled-device camera, the black curve is a Lorentzian fit. The blue curve corresponds to the spectrum after deconvoluting the spectral response of the detector (Lorentzian with FWHM $46 \mu \mathrm{eV}$ ) and therefore represents the quantum dot alone: the quantum dot photoluminescence spectrum has a FWHM of $36 \mu \mathrm{eV}$ for this particular quantum dot. $\mathbf{d}, \mathrm{A} \mathrm{g}^{2}$ measurement of a quantum-dot-in-nanowire (different quantum dot to a) using continuous wave excitation at $632.8 \mathrm{~nm}$. The count rate (right $y$-axis) was normalized to unity far from the dip at delay zero $t=0$ (left $y$-axis). The radiative lifetime $\tau$ of this quantum dot was measured to be 450 ps by recording the decay curve following pulsed excitation (see Supplementary Information). The black curve shows the convolution of the ideal two-level atom result, $g^{2}(t)=1-\exp (-|t| / \tau)$, with the response of the detectors (Gaussian with FWHM $0.62 \mathrm{~ns}$ ) and describes the data extremely well. The blue curve shows the two-level atom response only.

creates electrons and holes which relax rapidly into the quantum dot levels. In contrast, in the current quantum-dot-in-nanowire system, Fig. 1a, GaAs forms the lowest energy band edges, Fig. 1b, and acts as the ultimate sink for photoexcited carriers. Furthermore, the $2.5 \mathrm{~nm} 60 \% \mathrm{Al}$ shell at first sight poses a rather ineffective barrier for the quantum dot-confined electrons.

To understand the two-level-like emission features at energies above the GaAs band gap, we have studied theoretically quantum confinement in these large scale nanostructures. The two state-ofthe-art approaches used are the empirical pseudopotential method for a quantum-dot-in-nanowire of more than a half-million atoms, and density functional theory for a quantum wire of more than ten thousand atoms. For the empirical pseudopotential calculations, the computational cell, Fig. 2 e, contains the $10 \% \mathrm{Al}$ quantum dot with a square base in the (121) plane, of height $9.0 \mathrm{~nm}$, and four facets (112), (211), (121), and (212) to complete the pyramid; the $60 \% \mathrm{Al}$ barrier, parallel to the quantum dot facets, having a thickness of $2.5 \mathrm{~nm}$; the $30 \% \mathrm{Al}$ layer; and the GaAs substrate, parallel to the quantum dot (121) plane containing 14 monolayers of GaAs. The total number of atoms in the computational cell is 511,104 . We solve the atomistic Schrödinger equation using as potential the superposition of the atomistic pseudopotentials of $\mathrm{Ga}, \mathrm{Al}$ and $\mathrm{As}$ at the corresponding lattice sites given by the structure, in a basis of the linear combination of bulk bands ${ }^{22}$ using the folded spectrum method ${ }^{23}$, allowing the eigensolutions to be obtained in a physically interesting energy window. Excitonic effects are calculated from the single-particle states with a configuration interaction calculation. We find that the highest occupied molecular orbital and lowest unoccupied molecular orbital of the whole system reside indeed on the GaAs substrate (correspondingly, in the nanowire core), as shown in Fig. 5a. This supports our assignment of the broadband emission at $1.51 \mathrm{eV}$ to the nanowire GaAs core. The 67 lowest hole levels of the system as a whole $\left(\mathrm{h}_{0}-\mathrm{h}_{66}\right)$ are localized in the GaAs. The first hole state confined to the quantum dot is $h_{\mathrm{QD}}$ and corresponds to hole state $\mathrm{h}_{67}$ with energy $95 \mathrm{meV}$ below the bulk GaAs valence band edge ( $67 \mathrm{meV}$ below the system Highest occupied molecular orbital state $h_{0}$ ), as shown in Fig. $1 b$. For the electron states, the lowest 19 electron levels of the entire system $\left(\mathrm{e}_{0}-\mathrm{e}_{18}\right)$ are localized on GaAs, and the first state confined to the quantum dot is $\mathrm{e}_{\mathrm{OD}}$, corresponding to $\mathrm{e}_{19}$ with energy $317 \mathrm{meV}$ above the bulk GaAs conduction band edge (182 meV above the system lowest unoccupied molecular orbital state $\mathrm{e}_{0}$ ), as shown in Fig. $1 \mathrm{~b}$. The wavefunctions of states $\mathrm{e}_{0}, \mathrm{~h}_{0}, \mathrm{e}_{\mathrm{QD}}$ and $\mathrm{h}_{\mathrm{QD}}$ are shown in Fig. 5a. The calculated emission energy of the quantum dot-localized states is $1.902 \mathrm{eV}(652 \mathrm{~nm})$, red-shifted from the single-particle transition energy $1.932 \mathrm{eV}$ by excitonic effects.

Turning next to the density functional theory calculations, we have studied a quantum wire bound by polar and non-polar interfaces, thereby taking into account both internal electric fields and charge reorganization effects. We model the $10 \% \mathrm{Al}$ quantum dot by an infinite [1 $2 \overline{1} 1$-oriented wire embedded in a $60 \% \mathrm{Al}$ matrix with thickness either $1.4 \mathrm{~nm}$ (5,000 atom system) or $2.5 \mathrm{~nm}(12,168$ atom system), as shown in Fig. 5b-d. In both cases, the interface polarity was found to be insufficient to induce charge separation. We observe clearly defined quantum dot-localized states close to the valence and conduction band edges (Fig. 5b-d). Using the experimental bulk GaAs band gap for the core region where the edge states are dominant, but otherwise retaining our computed band offsets, we predict transitions between these levels at $1.94 \mathrm{eV}$ $(640 \mathrm{~nm})$, in broad agreement with both the unscreened calculation 
a
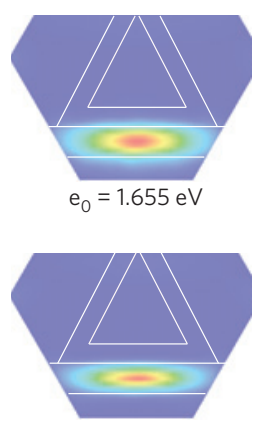

$h_{0}=-0.028 \mathrm{eV}$
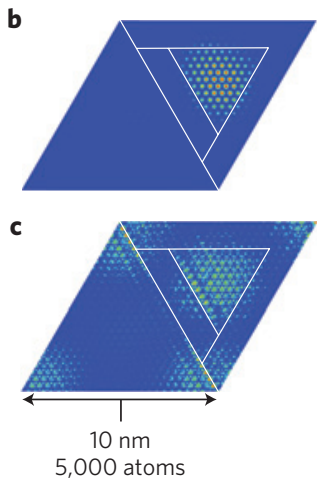

5,000 atoms

Figure 5 | Atomistic calculations of electronic states in a quantum-dotin-nanowire system. a, Pseudopotential eigenstate densities for a $10 \% \mathrm{Al}$ content $\mathrm{Al}_{x} \mathrm{Ga}_{1-x}$ As quantum dot, $9 \mathrm{~nm}$ high, surrounded by a $2.5 \mathrm{~nm}$ thickness $60 \% \mathrm{AlGaAs}$ barrier embedded in an $30 \% \mathrm{AlGaAs}$ matrix and sitting on a pure $\mathrm{GaAs}$ substrate ( $4 \mathrm{~nm}$ thickness in computational cell). Plotted are the lowest energy conduction and valence states ( $e_{0}$ and $h_{0}$, respectively) and the lowest energy quantum dot-bound conduction and valence states ( $e_{19}$ and $h_{67}$, respectively). b-d, The localized Kohn-Sham states nearest the band edges, computed using self-consistent linear-scaling density functional theory, and viewed along the [111] axis. b. Highest localized valence state, $0.07 \mathrm{eV}$ below the band edge, for a 5,000 atom model system $\left(10.0 \times 10.0 \times 1.3 \mathrm{~nm}^{3}\right.$, fully periodic), simulating the thin $(1.4 \mathrm{~nm}) 60 \% \mathrm{Al}$ barrier between the $10 \% \mathrm{Al}$ quantum dot with a pure GaAs nanowire shell. c, Corresponding conduction state, located at $0.36 \mathrm{eV}$ above the band edge. d, As c, but for a 12,168 atom system $\left(15.6 \times 15.6 \times 1.3 \mathrm{~nm}^{3}\right.$, fully periodic, with a barrier of width $2.5 \mathrm{~nm}) ; 0.27 \mathrm{eV}$ above the conduction edge. $\mathbf{b}$-d were produced using VESTA 3 (ref. 41).

and the experimental observation. We find that the thin $1.4 \mathrm{~nm}$, $60 \% \mathrm{Al}$ barrier is sufficient to provide quantum confinement irrespective of the polarity of the facets. Hence, both the atomistic pseudopotential of the quantum-dot-in-nanowire and density functional theory (DFT) of a charge-reorganized nanowire with the same cross-section predict states with both electrons and holes confined to the quantum dot.

In summary, we report the self-assembly of a high-quality quantum-dot-in-nanowire system using two basic components, GaAs and AlAs. Self-assembly is not strain driven. Instead, it proceeds by the different adatom mobility of $\mathrm{Ga}$ and $\mathrm{Al}$ on the host substrate. This mechanism may well be effective with other semiconductor materials (see Supplementary Information). The shape and composition of the quantum dots has been determined by high-resolution, atom-selective electron microscopy. Individual quantum dots are bright and spectrally pure emitters of highly anti-bunched light even with non-resonant excitation and even, furthermore, when they are positioned just a few nanometres from the nanowire surface. The operating principle is that both electron and hole states involved in the transition lie above the respective band edges of the nanowire itself, a point we understand quantitatively using atomistic calculations of the electronic states. As well as applications as single-photon sources, an immediate possibility is the application of these quantum dots as nano-sensors and in forging a coupling between the optical and mechanical properties. By adjusting the core and shell diameters of the nanowires, the quantum dot emission can be efficiently funnelled into a waveguide mode in the nanowire. Furthermore, there are easy routes to embedding the quantum dots in a radial $\mathrm{p}-\mathrm{n}$ junction ${ }^{24}$, opening up applications involving not just quantum light creation but also charge control ${ }^{25}$, single-photon detection ${ }^{26}$ and $\operatorname{spin}^{27,28}$.

\section{Methods}

Nanowire growth. The nanowires were grown using a DCA P600 MBE machine. The nanowire core structures were obtained under rotation at 7 r.p.m. at a temperature of $640^{\circ} \mathrm{C}$ under a flux of Ga equivalent to a planar growth rate of $0.03 \mathrm{~nm} \mathrm{~s}^{-1}$ and a V/III ratio of 60 . The conditions were then switched from axial to radial growth by increasing the As pressure and reducing the substrate temperature. A $50 \mathrm{~nm} \mathrm{Al} \mathrm{Aa}_{1-x}$ As shell was grown with $\mathrm{Al}$ compositions $x=0.33,0.51$ and 0.70 and capped with $5 \mathrm{~nm} \mathrm{GaAs}$ to prevent oxidation. One sample was grown with alternating layers of GaAs/AlGaAs at $x=0.33$.

Electron microscopy. Cross-sections of the nanowires perpendicular to the growth axis were prepared by mechanical polishing and ion milling. 3D atomic models were obtained using the Rhodius software package ${ }^{29}$, which allows complex atomic models to be created, including nanowire-like heterostructures ${ }^{30}$ High-angle annular dark-field scanning transmission electron microscopy analyses were performed in an aberration-corrected probe FEI Titan $60-300 \mathrm{keV}$ microscope. EDX analysis was performed using a FEI Tecnai OSIRIS microscope operated at $200 \mathrm{kV}$ using the Super-X (0.9 radian collection angle) detector and Bruker Esprit software.

Optical spectroscopy. The nanowires were transferred to a fresh silicon substrate and subsequently probed side-on. Cathodoluminescence was realized in an adapted scanning electron microscope ${ }^{31}$. Photoluminescence measurements were made with a confocal optical microscope with sample scanning, exciting with a low-power $\mathrm{HeNe}$ laser at $632.8 \mathrm{~nm}$ or $\mathrm{Ar}^{+} \mathrm{Kr}^{+}$laser at 488 and $514 \mathrm{~nm}$. The photoluminescence was dispersed with a monochromator-array detector system. Photoluminescence in a bandwidth of $0.5 \mathrm{~nm}$ was sent to a Hanbury Brown-Twiss photon coincidence set-up with two nominally identical silicon avalanche photodiodes. The pulsed measurements (see Supplementary Information) were performed with a Q-switched semiconductor laser diode at $635 \mathrm{~nm}$, using time-correlated single-photon counting to record the decay curve (Supplementary Information).

Pseudopotential calculations. The screened atomic potentials are adjusted by the empirical pseudopotentials method to correct for the DFT errors in band gaps, effective masses, inter-valley splittings and band offsets ${ }^{32}$. The single-particle problem is solved numerically in a plane-wave basis, including spin-orbit $(340 \mathrm{meV}$ for GaAs), using the folded spectrum method $^{33}$, which allows eigensolutions to be obtained in a physically interesting energy window (about $300 \mathrm{meV}$ from band edges) rather than at all energies. The calculation is carried out with a fixed potential without iterating to self-consistency.

Self-consistent DFT calculations. Using linear-scaling DFT (ref. 34), as implemented in the ONETEP method ${ }^{35-37}$, which captures charge redistribution effects both efficiently and accurately ${ }^{38,39}$, we have performed simulations on structures containing 5,000 and 12,168 atoms (one of the largest fully self-consistent calculations ever performed). The 5,000 atom calculation was carried out by iteratively refining a compact real-space Wannier basis with respect to a primary plane-wave basis, whereas in the 12,168 atom calculation the real-space basis was pre-optimized for isolated atoms, and thereafter fixed. In order to capture both the polar [121] and non-polar facets of the observed quantum-dots-in-nanowires, our simulation cells consist of fully-periodic slabs ( $1.3 \mathrm{~nm}$ thick, along the [1iㅣ] direction) of an effective [1리] wire. Simulations at the interface with the GaAs outer shell (Fig. 5) and with the $\mathrm{Al}_{0.3} \mathrm{Ga}_{0.7}$ As nanowire core were separately carried out (see Supplementary Information), using norm-conserving pseudopotentials, the local-density approximation ${ }^{40}$, and random alloying at each stated concentration.

Received 26 June 2012; accepted 21 December 2012; published online 3 February 2013

\section{References}

1. Shields, A. J. Semiconductor quantum light sources. Nature Photon. 1, 215-223 (2007) 
2. Borgstrom, M. T., Zwiller, V., Muller, E. \& Imamoglu, A. Optically bright quantum dots in single nanowires. Nano Lett. 5, 1439-1443 (2005).

3. Claudon, J. et al. A highly efficient single-photon source based on a quantum dot in a photonic nanowire. Nature Photon. 4, 174-177 (2010).

4. Bulgarini, G. et al. Spontaneous emission control of single quantum dots in bottom-up nanowire waveguides. Appl. Phys. Lett. 100, 121106 (2012).

5. Reimer, M. E. et al. Bright single-photon sources in bottom-up tailored nanowires. Nature Commun. 3, 737 (2012)

6. Heinrich, J. et al. Single photon emission from positioned GaAs/AlGaAs photonic nanowires. Appl. Phys. Lett. 96, 211117 (2010).

7. Uccelli, E., Arbiol, J., Morante, J. R. \& Fontcuberta i Morral, A. InAs quantum dot arrays decorating the facets of GaAs nanowires. ACS Nano 4, 5985-5993 (2010).

8. Bounouar, S. et al. Ultrafast room temperature single-photon source from nanowire-quantum dots. Nano Lett. 12, 2977-2981 (2012).

9. Agarwal, R. \& Lieber, C. M. Semiconductor nanowires: Optics and optoelectronics. Appl. Phys. A 85, 209-215 (2006).

10. Kelzenberg, M. D. et al. Enhanced absorption and carrier collection in Si wire arrays for photovoltaic applications. Nature Mater. 9, 239-244 (2010).

11. Moreau, E. et al. Single-mode solid-state single photon source based on isolated quantum dots in pillar microcavities. Appl. Phys. Lett. 79, 2865-2867 (2001).

12. Moskalenko, E. S., Larsson, M., Schoenfeld, W. V., Petroff, P. M. \& Holtz, P. O. Carrier transport in self-organized InAs/GaAs quantum-dot structures studied by single-dot spectroscopy. Phys. Rev. B 73, 155336 (2006).

13. Dalgarno, P. A. et al. Hole recapture limited single photon generation from a single n-type charge-tunable quantum dot. Appl. Phys. Lett. 92, 193103 (2008)

14. Colombo, C., Spirkoska, D., Frimmer, M., Abstreiter, G. \& Fontcuberta i Morral, A. Ga-assisted catalyst-free growth mechanism of GaAs nanowires by molecular beam epitaxy. Phys. Rev. B 77, 155326 (2008).

15. Heigoldt, M. et al. Long range epitaxial growth of prismatic heterostructures on the facets of catalyst-free GaAs nanowires. J. Mater. Chem. 19, 840-848 (2009).

16. Fontcuberta i Morral, A. et al. Prismatic quantum heterostructures synthesized on molecular-beam epitaxy GaAs nanowires. Small 4, 899-903 (2008).

17. Biasiol, G. \& Kapon, E. Mechanisms of self-ordering of quantum nanostructures grown on nonplanar surfaces. Phys. Rev. Lett. 81, 2962-2965 (1998).

18. Biasiol, G., Gustafsson, A., Leifer, K. \& Kapon, E. Mechanisms of self-ordering in nonplanar epitaxy of semiconductor nanostructures. Phys. Rev. B 65, 205306 (2002).

19. Steinke, L. et al. Nanometer-scale sharpness in corner-overgrown heterostructures. Appl. Phys. Lett. 93, 193117 (2008).

20. Skold, N. et al. Phase segregation in AlInP shells on GaAs nanowires. Nano Lett. 6, 2743-2747 (2006)

21. Dalgarno, P. A. et al. Coulomb interactions in single charged self-assembled quantum dots: Radiative lifetime and recombination energy. Phys. Rev. B 77, 245311 (2008).

22. Wang, L. W. \& Zunger, A. Linear combination of bulk bands method for large-scale electronic structure calculations on strained nanostructures. Phys. Rev. B 59, 15806-15818 (1999).

23. Franceschetti, A., Fu, H., Wang, L. W. \& Zunger, A. Many-body pseudopotential theory of excitons in InP and CdSe quantum dots. Phys. Rev. B 60, 1819-1829 (1999).

24. Colombo, C., Heiß, M., Grätzel, M. \& Fontcuberta i Morral, A. Gallium arsenide p-i-n radial structures for photovoltaic applications. Appl. Phys. Lett. 94, 173108 (2009)

25. Bjork, M. T. et al. Few-electron quantum dots in nanowires. Nano Lett. 4, 1621-1625 (2004)

26. Hayden, O., Agarwal, R. \& Lieber, C. M. Nanoscale avalanche photodiodes for highly sensitive and spatially resolved photon detection. Nature Mater. 5, 352-356 (2006).

27. Van Weert, M. H. M. et al. Selective excitation and detection of spin states in a single nanowire quantum dot. Nano Lett. 9, 1989-1993 (2009).

28. Witek, B. J. et al. Measurement of the g-factor tensor in a quantum dot and disentanglement of exciton spins. Phys. Rev. B 84, 195305 (2011).

29. Bernal, S. et al. The interpretation of HREM images of supported metal catalysts using image simulation: profile view images. Ultramicroscopy $\mathbf{7 2}$, 135-164 (1998).

30. Arbiol, J. et al. Optimization of tin dioxide nanosticks faceting for the improvement of palladium nanocluster epitaxy. Appl. Phys. Lett. 80, 329-331 (2002).
31. Heiss, M. et al. Catalyst-free nanowires with axial $\operatorname{In}_{x} \mathrm{Ga}_{1-x} \mathrm{As} / \mathrm{GaAs}$ heterostructures. Nanotechnology 20, 075603 (2009).

32. Luo, J.-W., Bester, G. \& Zunger, A. Atomistic pseudopotential calculations of thickness-fluctuation GaAs quantum dots. Phys. Rev. B 79, 125329 (2009).

33. Wang, L. W. \& Zunger, A. Solving Schrodinger's equation around a desired energy-Application to silicon quantum dots. J. Chem. Phys. 100, 2394-2397 (1994).

34. Bowler, D. R. \& Miyazaki, T. O(N) methods in electronic structure calculations. Rep. Prog. Phys. 75, 036503 (2012)

35. Skylaris, C. K., Haynes, P. D., Mostofi, A. A. \& Payne, M. C. Introducing ONETEP: Linear-scaling density functional simulations on parallel computers. J. Chem. Phys. 122, 084119 (2005).

36. Hine, N. D. M., Haynes, P. D., Mostofi, A. A. \& Payne, M. C. Linear-scaling density-functional simulations of charged point defects in $\mathrm{Al}_{2} \mathrm{O}_{3}$ using hierarchical sparse matrix algebra. J. Chem. Phys. 133, 114111 (2010).

37. Hine, N. D. M., Haynes, P. D., Mostofi, A. A., Skylaris, C-K. \& Payne, M. C. Linear-scaling density-functional theory with tens of thousands of atoms: Expanding the scope and scale of calculations with ONETEP. Comput. Phys. Commun. 180, 1041-1053 (2009).

38. Avraam, P. W., Hine, N. D. M., Tangney, P. \& Haynes, P. D. Fermi-level pinning can determine polarity in semiconductor nanorods. Phys. Rev. B 85, 115404 (2012).

39. O’Regan, D. D., Payne, M. C. \& Mostofi, A. A. Generalized Wannier functions: A comparison of molecular electric dipole polarizabilities. Phys. Rev. B 85, 193101 (2012).

40. Perdew, J. P. \& Zunger, A. Self-interaction correction to density-functional approximations for many-electron systems. Phys. Rev. B 23, 5048-5079 (1981).

41. Momma, K. \& Izumi, F. VESTA 3 for three-dimensional visualization of crystal, volumetric and morphology data. J. Appl. Crystallogr. 44, 1272-1276 (2011).

\section{Acknowledgements}

A.F.i.M. acknowledges funding from ERC through the UpCon grant and SNF through Grant No. 134506. Both A.F.i.M. and R.J.W. acknowledge support from NCCR QSIT. The Phantoms Foundation is acknowledged for sponsoring B.K.'s visit to Lund University. This work was supported by the Spanish MICINN Projects MAT2010-15138, CSD2009-00013 and CSD2009-00050. J.A. and J.R.M. acknowledge Generalitat de Catalunya 2009-SGR-770, NanoAraCat and XaRMAE and European RDF support. The authors acknowledge F. J. Belarre for the making of the TEM cross-sections; A.G. thanks L. Samuelson and the Swedish Research Council for support. D.D.O. acknowledges N. D. M. Hine and the ONETEP developers' group for discussions and software support, and the EPFL HPC service for generous provision of computing resources. The work carried out by J.W.L. and A.Z. was funded by the US Department of Energy, Office of Science, Basic Energy Science, Materials Sciences and Engineering under contract number DE-AC36-08GO28308 to NREL and CU Boulder.

\section{Author contributions}

M.H., E.R-A. and A.F.i.M. grew the samples. J.A., J.R.M. and C.M. performed HAADF STEM and EELS analysis. S.C-B. and M.C. mapped the composition of the quantum dots by EDX. J.A. and S.C-B. worked on the atomic modelling of the quantum dots. A.G. and B.K. measured the cathodoluminescence. M.H., Y.F., G.W., A.V.K., J.H. and R.J.W. participated in the optical spectroscopy studies. D.D.O. and N.M. performed density functional theory calculations. J.W.L. and A.Z. performed the empirical pseudopotential calculations. A.F.i.M. conceived and designed the experiments, and together with R.J.W., A.Z. and N.M. supervised the project. Y.F. made the figures and artwork. A.F.i.M., R.J.W., Y.F., A.Z., D.D.O., N.M. and J.A. wrote and edited the manuscript. All authors discussed the results and commented on the manuscript.

\section{Additional information}

Supplementary information is available in the online version of the paper. Reprints and permissions information is available online at www.nature.com/reprints. Correspondence and requests for materials should be addressed to A.F.i.M.

\section{Competing financial interests}

The authors declare no competing financial interests. 